\title{
Interference-aware multipath routing in wireless mesh network
}

\author{
Faiza lqbal $^{1 *}$, Muhammad Younus Javed ${ }^{1}$ and Anjum Naveed ${ }^{2}$
}

\begin{abstract}
The inherent mesh infrastructure of IEEE 802.11-based wireless mesh networks provides added support to construct multiple robust paths. However, based on geometric locations of wireless nodes, neighborhood interference and channel contention impose challenges on multipath routing schemes. A large body of research has been carried out to maximize aggregate end-to-end throughput using multipath routing; however, interference has not been accurately modeled in majority of the work. Based on the relative location of transmitters and receivers, information asymmetric non-coordinated interference introduces bottleneck links and significantly reduces the aggregate throughput. The impact of this interference is even observed on links several hops away. In this paper, multipath routing is integrated with topology control to manage such multilevel asymmetric interference. An optimization model has been presented with an objective to achieve optimized end-to-end throughput using multiple available paths, considering coordinated and asymmetric non-coordinated interference. The goal of this research is to develop a multipath routing strategy which can achieve better end-to-end throughput by purging badly affected asymmetric non-coordinated interfering links during path construction procedure. The proposed model and routing strategy have been tested through extensive simulations. The results clearly exhibit the efficacy of the proposed approach, which achieves better aggregate end-to-end throughput compared to existing multipath routing schemes.
\end{abstract}

Keywords: Wireless mesh networks; Linear programming; Multipath routing

\section{Introduction}

IEEE 802.11-based wireless mesh networks (WMN) have emerged as a promising architecture to provide lastmile Internet connectivity to fixed and mobile users. The architecture of WMN usually comprises mesh clients, mesh routers, and gateways. Mesh routers connect mesh clients with gateways using multihop wireless links. Several deployment scenarios of WMN are in practical use including deployment for coverage enhancement, in-door deployment where Internet cabling is difficult, community-wide deployment such as university campuses, and deployment in disaster-struck areas $[1,2]$.

Despite a number of worldwide WMN deployments and projects with communications based on WMN [3-6], many research challenges exist that, if addressed,

\footnotetext{
*Correspondence: faizaiqbal@ceme.nust.edu.pk

${ }^{1}$ College of EME, National University of Sciences and Technology (NUST),

Islamabad, Pakistan

Full list of author information is available at the end of the article
}

can significantly improve the achievable throughput. In particular, given the broadcast nature of the wireless medium, interference has a significant impact on the capacity of wireless links. A combination of accurate interference estimation and interference avoidance/mitigation techniques is mandatory for acceptable network performance. Accurate estimation of interference can lead to interference avoidance by exploiting the inherent redundant infrastructure of WMN through efficient routing and low-interference path selection.

Over the past decade, a number of unipath and multipath routing schemes and protocols have been proposed [7-22]. Significant body of research has used protocol model $[11,17,22]$, physical model $[17,23]$, or measurement-based models [9,24-31] for interference estimation. However, none of these models and metrics accurately capture the impact of interference. Consequently, the selected routing paths do not lead to optimal end-to-end throughput. The basic reason for inaccuracy is the underlying assumption that all interfering links have similar impact. In practice, this assumption

\section{Springer}

(C) 2014 lqbal et al: licensee Springer. This is an Open Access article distributed under the terms of the Creative Commons

Attribution License (http://creativecommons.org/licenses/by/2.0), which permits unrestricted use, distribution, and reproduction in any medium, provided the original work is properly credited. 
does not hold and the relative location of the interfering links leads to different impacts of interference. Based on relative locations of transmitters and receivers, Garetto et al. [32] have categorized interfering links into four categories.

According to the authors, two links are said to be coordinated interfering links if the transmitters of links are within carrier sensing range of each other. These coordinated transmitters prevent each other from concurrent transmissions, resulting in negligible packet collisions. On the other hand, if transmitters of the two interfering links are not within carrier sensing range of each other, three additional categories of interference near hidden, far hidden, and information asymmetric are possible. We collectively refer to the interference caused by these categories as non-coordinated (non$\mathrm{CO}$ ) interference. In case of non-coordinated interfering links, the two transmitters cannot sense the transmissions of each other. This results in increased probability of collisions. Particularly, among non-coordinated interference, information asymmetric interference is the most detrimental in terms of unfair channel sharing and end-to-end throughput performance [32]. The asymmetry of the channel view of the two information asymmetric interfering links allows one link to achieve its maximum throughput while the other link starves. Consequently, bottleneck links are introduced in the network, leading to reduced end-to-end throughput. Therefore, an efficient routing scheme is required, which considers coordinated as well as asymmetric noncoordinated interactions of transmitters and receivers during path construction procedure to avoid such bottleneck links.

Recent studies have considered non-coordinated interactions during routing decisions. Salonidis et al. [33] have proposed a routing metric AVAIL based on the maximum available bandwidth between source and destination. Authors have proposed the use of fraction of busy time and packet loss probability to identify high-throughput paths. Similarly, Razak et al. [34] have proposed a MAC Interaction Aware Routing metric (MIAR), which considers non-coordinated MAC interactions while constructing a route. Both metrics are focused on creating single path for routing flows and ignore the advantage of redundant mesh infrastructure. Furthermore, it is known that information asymmetric interference not only affects links within carrier sensing range but also affects the throughput of links several hops away [35]. To the best of our knowledge, such multilevel impact of information asymmetric interference has not been addressed by existing routing schemes.

The research study in this paper highlights the adverse impact of information asymmetric interference on end- to-end throughput in wireless networks. The advantage of selecting a subset of quality paths over all available paths or using single path per flow is also explained using motivational examples (Section 2). The examples show that purging certain links from network topology reduces the number of available alternate paths for the flows; however, the quality of remaining paths is improved, resulting in better end-to-end throughput. Primary objective of this research is to show the effectiveness of combining the topology control to mitigate non-coordinated interference with multipath routing in wireless mesh networks to achieve significantly improved aggregate end-to-end throughput. Topology control is used to prune the links from the network that can lead to multilevel information asymmetric interference. Consequently, bottleneck links can be avoided in the network. In Section 3, an optimization model has been proposed for joint topology control and multipath routing. The model aims at selecting a subset of quality paths for each flow such that the aggregate end-to-end throughput is maximized. Selection of paths is constrained by per link capacity and interference at each link. The effectiveness of the model in accurately predicting the end-to-end throughput is shown through simulations.

Adaptive Multipath Routing with Topology Control (AMRTC) scheme has been proposed in Section 4. AMRTC is a two-phase centralized scheme. The first phase of AMRTC performs topology control by identifying and pruning the multilevel non-coordinated interfering links. Resultant topology control graph is used in the second phase where maximum available residual capacity paths to satisfy the flow demands are selected. Extensive simulation-based evaluation is carried out to study the effectiveness of the proposed scheme. The results show that AMRTC can effectively mitigate the impact of coordinated as well as non-coordinated interference and can achieve good performance under different scenarios. The results of the evaluation have been presented in Section 5. Section 6 concludes the study.

\section{Motivation}

This section highlights the significance of joint topology control and multipath routing in improving the end-to-end throughput of multihop wireless networks. A number of topological scenarios have been considered. The topologies are simulated using OPNET Modeler 14.0. The nodes are equipped with IEEE $802.11 \mathrm{~b}$ radios which are operating on a common channel. The transmission range $\left(R_{\mathrm{tr}}\right)$ and carrier sensing range $\left(R_{\mathrm{cs}}\right)$ of the nodes are assumed to be fixed such that $R_{\mathrm{cs}} \approx$ $1.8 R_{\mathrm{tr}}$. CBR traffic is generated for experiments in this 
section with packets of 512 B. Figures 1a and 2a represent wireless single and multihop topologies considered for this section. The solid-headed arrows represent the directed links, while dotted lines connect nodes within carrier sensing range (in addition to connected nodes).

\subsection{Reducing interference dependencies and bottlenecks using topology control}

This section highlights the adverse impact of noncoordinated interference on end-to-end throughput. Consider the topology represented by Figure 1a. The nodes are positioned in such a way that directed link $l_{1}$ is observing asymmetric non-CO interference from link $l_{2}$, while $l_{2}$ is under asymmetric non-CO interference of link $l_{3}$. The same multilevel interference exists between $l_{3}$ and $l_{4}$, producing a chain of multilevel interference dependencies.

Figure $1 \mathrm{~b}$ shows the throughput of single-hop flows operating on the four links of Figure 1a. The graph shows the throughput of four flows when the offered load of flow $f_{4}$ on link $l_{4}$ is gradually increased while the offered load of flows $f_{1}, f_{2}$, and $f_{3}$ (operating on links $l_{1}, l_{2}$, and

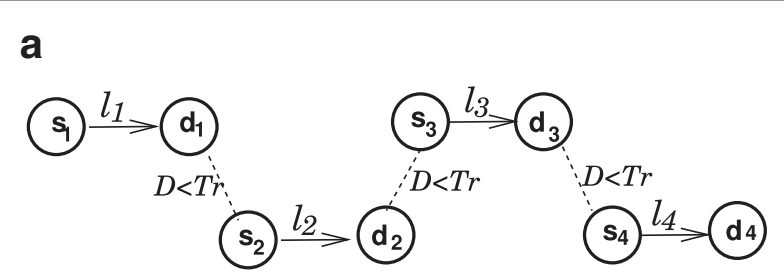

b

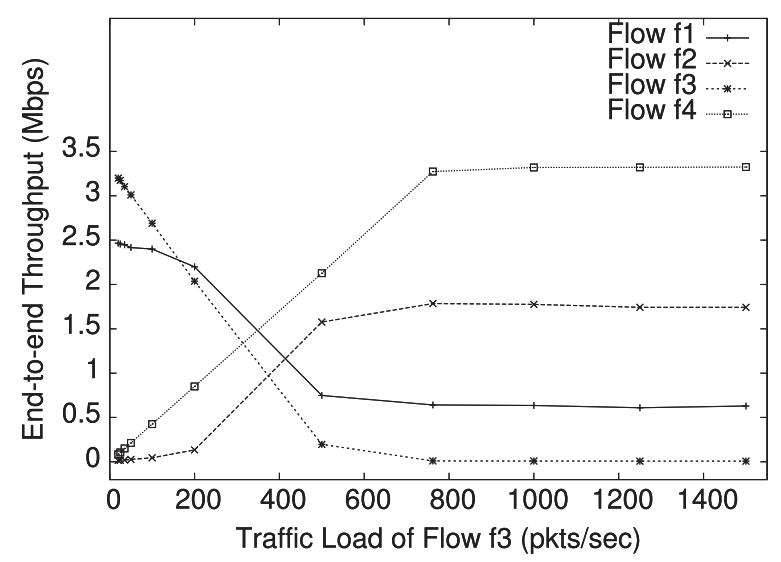

Figure 1 Impact of non-coordinated interference dependencies. (a) Single-hop topology, showing multilevel non-CO interference dependencies. ( $\mathrm{D}$ is the distance while $\mathrm{Tr}$ is transmission range). (b) End-to-end throughput of single-hop topology, showing the impact of multilevel asymmetric non-CO interference dependencies. $l_{3}$, respectively) are kept constant. Observe that as the load on $l_{4}$ is increased from 0.212 to $3.232 \mathrm{Mbps}$, the achievable throughput of link $l_{1}$ (which is outside carrier sensing range of $l_{4}$ ) decreases from 2.465 to 0.628 Mbps. This is due to the presence of multilevel information asymmetric non-CO interference, the impact of which is observed on even remote links. Also, observe that the throughput achieved by links $l_{1}$ and $l_{3}$ is significantly lower when link $l_{4}$ operates in a saturated condition. If the path of a particular flow includes links like $l_{1}$ or $l_{3}$, the end-to-end throughput will severely be affected by load on link $l_{4}$. In this paper, we propose pruning the links causing multilevel non-coordinated interference through topology control. This form of topology control reduces the number of available alternate paths for routing. However, the better quality of remaining paths helps in improving overall aggregate end-to-end throughput.

\subsection{Impact of number of paths on aggregate throughput}

The key consideration in multipath routing is the selection of number of parallel paths per flow. Research shows that using too many paths is not useful [36]. Nasipuri et al. [7] have analytically proved that the performance advantage of using more than two alternate routes is usually minimal. A simple experiment shows the effect of number of paths on throughput of multihop flows.

Consider the multihop topology of Figure 2a. The throughput of flow $f_{1}\left(s_{1}, d_{1}\right)$ over multiple paths is compared by increasing its offered traffic load from 20 to $1,500 \mathrm{pkts} / \mathrm{s}$ (packet size of $512 \mathrm{~B}$ ). Figure $2 \mathrm{~b}$ shows the average throughput of flow $f_{1}$ as a function of offered traffic load. The graph shows that under unsaturated conditions, the number of paths does not impact the throughput. However, under saturated traffic load (200 pkts/s), $f_{1}$ achieves the maximum throughput of 0.819 Mbps when routed using two paths. On the other hand, $f_{1}$ achieves a maximum throughput of $0.674,0.706,0.379$, and $0.376 \mathrm{Mbps}$ when routed using single, three, four and five paths, respectively. Note that not all paths are linkdisjoint in the case of four and five paths. This shows that increasing the number of paths increases interference on the links, which results in reduced aggregate throughput.

We now show that the quality of paths varies because of varying level of coordinated and non-coordinated interferences. Therefore, selecting any pair of alternate paths will not always result in improved throughput.

\subsection{Selection of good quality paths}

While opting to route on multiple available paths, usually maximal disjoint paths are selected between a source- 
a

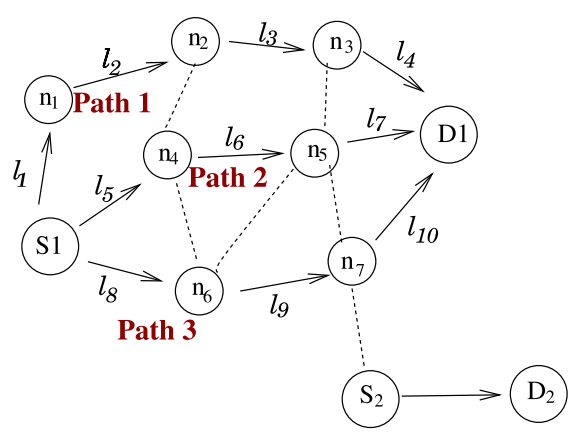

b

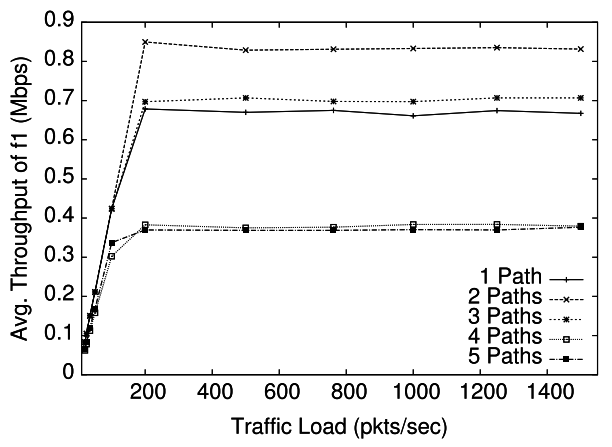

\section{C}

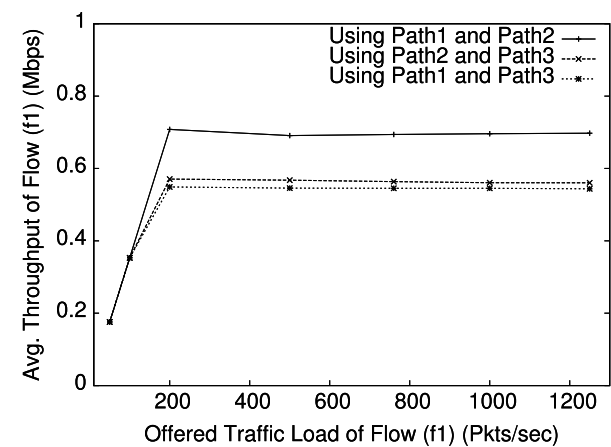

Figure 2 Effectiveness of using fewer good quality paths vs. using all alternate paths. (a) Multihop topology, showing multiple link-disjoint and node-disjoint paths observing non-CO interference. (b) Impact of increasing number of parallel paths on average flow throughput. (c) Impact of using fewer 'quality' paths on average flow throughput.

destination pair. Given a directed graph $G(V, E)$, having source-destination pair $\left(s_{i}, d_{i}\right)$, a path $p_{i} \in P\left(f\left(s_{i}, d_{i}\right)\right)$ is defined as fully node/link disjoint if it does not share node/link with any other path $p_{j}$ traversed by the flow $f\left(s_{i}, d_{i}\right)$. The path $p_{i}$ is defined as maximally node/link disjoint if it shares a minimum number of nodes/links with other paths.

Consider again multihop topology of Figure 2a. Threenode-disjoint and link-disjoint paths (Path1 $\left(l_{1}, l_{2}, l_{3}, l_{4}\right)$, Path $2\left(l_{5}, l_{6}, l_{7}\right)$ and Path3 $\left.\left(l_{8}, l_{9}, l_{10}\right)\right)$ are available for flow $f_{1}\left(s_{1}, d_{1}\right)$. A single-hop flow $f_{2}\left(s_{2}, d_{2}\right)$ operating under saturated traffic load is creating interference on links of Path 2 and Path 3 of flow $f_{1}$. Figure 2c shows aggregate throughput of flow $f_{1}$ when different subsets of paths are selected for routing. It is obvious that not all pairs of paths lead to the same throughput. The throughput is significantly lower when Path 2 and (or) Path3 is chosen. This is because link $l_{9}$ of Path 3 is experiencing non-coordinated interference from flow $f_{2}$. Similarly, link $l_{5}$ of Path 2 is experiencing non-coordinated interference from link $l_{9}$. Consequently, a chain similar to that of Figure 1a is formed, resulting in a bottleneck link on
Path3. Therefore, ignoring $l_{9}$ during link selection phase of path construction procedure can eliminate the chain. This helps in improving effective capacity of $l_{5}$, causing Path2 to offer more flow capacity to flow $f_{1}$. Note that link pair $l_{4}, l_{1}$ is also non-coordinated pairs; however, this pair belongs to same path and none of the links operates in saturated mode. Therefore, the impact of interference in minimized, resulting in significant opportunities for link $l_{1}$.

The above examples illustrate that the approach of topology control for reducing non-coordinated interference and resulting in fewer but high-quality paths that can be used for multipath routing has merit. This approach can be used to significantly improve the aggregate end-toend throughput.

\section{Optimization model}

We now present the optimization framework of throughput maximization problem incorporating coordinated and non-coordinated interference constraints in multihop wireless mesh network. The model computes the upper 
bound on optimal throughput of a network with given traffic load and interference dependencies of neighboring nodes. We begin with a network model describing terminologies and assumptions.

\subsection{Network model}

Let directed graph $G(N, E)$, with $N$ being set of mesh nodes and $E \in N \times N$ being set of links, represent WMN. Let $d\left(n_{i}, n_{j}\right)$ represent the distance between $\left(n_{i}, n_{j}\right)$. An edge $e\left(n_{i}, n_{j}\right) \in E$ exists between $n_{i}$ and $n_{j}$ if $d_{i j} \leq R_{\mathrm{tr}}$. The maximum data capacity of link $e_{j}$ is given by $C_{e_{j}} \forall e_{j} \in E$. We assume that packets are not dropped at the nodes, if successfully transmitted over the links. Let $f_{i}$ represent the data demand of ith end-to-end flow in the network. Each flow may be routed through multiple sub-flows using multipath routing. Let $f_{i_{l}}$ represent a sub-flow of flow $f_{i}$. The notations used in optimization model are listed in Table 1.

\subsection{Interference model}

Let $e_{h}\left(n_{i}, n_{j}\right)$ denote a directed link between $n_{i}$ and $n_{j}$, where $n_{i}$ represents transmitter and $n_{j}$ represents receiver, then a directed link $e_{h} h_{h}\left(n_{i}, n_{j}\right)$ is an interfering link if $d\left(n_{i}, n \prime_{j}\right), d\left(n_{i}, n_{i}^{\prime}\right), d\left(n_{j}, n_{j}\right)$ or $d\left(n_{j}, n \prime_{i}\right) \leq R_{C S}$. According to Garetto et al. [32], interfering links can be classified into coordinated and non-coordinated interfering links, depending upon the Euclidean distance between $\left(n_{i}, n_{j}\right)$ and $\left(n / i, n_{j}\right)$. Two links $e_{h}\left(n_{i}, n_{j}\right)$ and $e^{\prime}{ }_{h}\left(n \prime_{i}, n \prime_{j}\right)$ are observing coordinated interference if $d\left(n_{i}, n_{i}\right) \leq R_{\mathrm{CS}}$, i.e., if the transmitters of the links are within the carrier sensing range of each other. On the contrary, two links $e_{h}\left(n_{i}, n_{j}\right)$ and $e^{\prime}{ }_{h}\left(n^{\prime}, n^{\prime} j\right)$ are said to be non-coordinated interfering links if $d\left(n_{i}, n_{i}\right)>R_{\mathrm{CS}}$ and $d\left(n_{i}, n_{j}^{\prime}\right) \leq R_{\mathrm{CS}}$, and/or $d\left(n \prime_{i}, n_{j}\right) \leq R_{\mathrm{CS}}$, and/or $d\left(n_{j}, n^{\prime} j\right) \leq R_{\mathrm{CS}}$. The inter-

\section{Table 1 List of notations}

\begin{tabular}{ll}
\hline Symbol & Description \\
\hline$N$ & Set of vertices/nodes \\
$E$ & Set of directed links \\
$G$ & Directed network topology graph \\
$G^{T}$ & Graph after topology control \\
$C_{e_{k}}$ & Capacity of link $e_{k} \in E$ \\
$f_{i}\left(n_{\mathrm{s}}->n_{\mathrm{d}}\right)$ & Flow between nodes $n_{\mathrm{s}}, n_{\mathrm{d}}$ \\
$f_{i_{i}}$ & Sub-flow of flow $f_{i}$ \\
$P\left(f_{i}\right)$ & Set of all links on path for flow $f_{i}$ \\
$L_{\mathrm{CO}}\left(e_{j}\right)$ & Set of coordinated links of $e_{j}$ \\
$L_{n C O}\left(e_{j}\right)$ & Set of non-coordinated links of $e_{j}$ \\
\hline
\end{tabular}

ference caused by coordinated links is logical (capacity is affected but there is no actual packet collision) as the coordinated transmitters sense the ongoing transmissions around their surroundings and avoid collisions through back off. On the other hand, the interference induced by non-coordinated links is physical because it results in collisions, packet losses, and unfair traffic distribution. In this paper, the interference model of Garetto et al. is used because of its accuracy in predicting the impact of interference.

\subsection{Assumptions}

The following assumptions have been made in the proposed optimization model and the proposed routing algorithm:

- The location of the routers is known to the centralized computation server. This information can be used to compute the coordinated and noncoordinated links for each pair of nodes.

- It is assumed that the flow demand of flows is known a priori. Although it is not possible to know the exact demand in advance, the flow demand can easily be estimated as the weighted average of MAC layer queue length and average data transmitted in previous intervals. This procedure has been used by researchers for flow demand estimation.

- We assume that a single interface is available per node. Therefore, all links operate on the same channel. It is easy to see that in case of multiradio multichannel WMN, the algorithm or the optimization model will not change. The only change will be in the number of interfering links, which will be selected as those links that physically belong to a set of interfering links and also operate on same channel. Note that dynamic channel assignment can also be accommodated by recomputing the routes. Further note that channel assignment schemes do not ensure interference elimination because of limited channels available. Consequently, all phases of optimization model and routing algorithm will be required as such.

\subsection{Linear program formulation}

We formulate joint topology control and multipath routing as linear optimization model. In this section, we explain the decision variable, constraints, and objective function of the model.

Decision variables The optimization model computes the normalized data flow of $f_{i}$ through link $e_{j}$. This is represented by decision variable $T_{f_{i}}\left(e_{j}\right)$ as shown below. The 
value of 0 for a given flow-link pair indicates that the link is not in path of that flow.

$$
T_{f_{i}}\left(e_{j}\right)=S_{i} \quad \text { where } 0 \leq S_{i} \leq 1
$$

\subsubsection{Routing constraints}

For any source-destination pair $n_{\mathrm{s}_{i}}, n_{\mathrm{d}_{i}} \in N$, the data produced by the source $n_{\mathrm{s}_{i}}$ must be consumed at the destination $n_{\mathrm{d}_{i}}$. This effectively means that although there are transmission errors and re-transmissions, there are no link failures. The following equation ensures that traffic generated by source of flow $f_{i}$ is equal to the traffic received by the destination of the flow:

$$
\Sigma_{n_{k} \in N} f_{i} * T_{f_{i}}\left(e_{\left(n_{s_{i}}, n_{k}\right)}\right)=\Sigma_{n_{p} \in N} f_{i} * T_{f_{i}}\left(e_{\left(n_{p}, n_{\mathrm{d}_{i}}\right)}\right) \quad \forall f_{i}
$$

Similarly, for all intermediate nodes (every node other than source and destination of a particular flow), the data received by the node for a specific flow should be equal to the data forwarded by that node for that particular flow. Thus we have

$$
\begin{aligned}
& f_{i} * T_{f_{i}}\left(e_{\left(n_{j}, n_{k}\right)}\right)=f_{i} * T_{f_{i}}\left(e_{\left(n_{k}, n_{p}\right)}\right) \\
& \forall n_{k} \in N \backslash\left\{v_{s_{i}}, v_{d_{i}}\right\}, \forall f_{i}, n_{j} \neq n_{p}
\end{aligned}
$$

The link-path constraint for a flow $f_{i}$ ensures that the flow cannot traverse a link which is not on any of its path. This leads us to the following constraint:

$$
\Sigma_{e_{j} \notin P\left(f_{i}\right)} f_{i} * T_{f_{i}}\left(e_{j}\right)=0 \quad \forall f_{i}
$$

\subsubsection{Interference and topology constraints}

All coordinated interfering links of link $e_{j}$ share the channel capacity with the link. These coordinated links are represented by set $L_{\mathrm{CO}}\left(e_{j}\right)$ in the network. The coordinated interference constraint thus ensures that sum of flow demands of all coordinated links must be less than or equal to the available effective capacity of link $e_{j}$.

$$
\Sigma_{f_{i}} f_{i} * T_{f_{i}}\left(e_{j}\right)+\Sigma_{f_{i}} \Sigma_{e_{k}} f_{i} * T_{f_{i}}\left(e_{k}\right) \leq C\left(e_{j}\right) \quad \forall e_{k} \in L_{\mathrm{CO}}\left(e_{j}\right)
$$

When a link $e_{j}$ experiences non-coordinated interference from another link, link $e_{j}$ gets the residual channel capacity. With set of links $L_{\mathrm{nCO}}\left(e_{j}\right)$ representing non-coordinated links of the link $e_{j}$, the noncoordinated interference constraint is given by following equation:

$$
\begin{aligned}
& \Sigma_{f_{i}} f_{i} * T_{f_{i}}\left(e_{j}\right) \leq C\left(e_{j}\right)-\Sigma_{f_{i}} \Sigma_{e_{k}} \operatorname{demand}_{f_{i}}\left(e_{k}\right) \\
& \forall e_{k} \in L_{\mathrm{nCO}}\left(e_{j}\right)
\end{aligned}
$$

The objective of topology control is to reduce interference chain dependencies. This is achieved by ensuring that data should not be routed through a link that is experiencing asymmetric non-coordinated interference and is itself inducing non-coordinated interference on other links in the network. Effectively, the bottleneck links are purged from the network, resulting in fewer quality paths. The constraint equation is given as

$$
\Sigma_{e_{k} \in P\left(f_{i}\right)} T_{f_{i}}\left(e_{k}\right)=0 \quad \forall f_{i}, \forall e_{k} \in L_{\mathrm{nCO}}\left(e_{j}\right) \exists e_{l} \in L_{\mathrm{nCO}}\left(e_{k}\right)
$$

\subsubsection{System constraints}

At any link $e_{j} \in E$, the maximum flow demand of all flows using that link is bound by the link capacity.

$$
\Sigma_{f_{i}} f_{i} * T_{f_{i}}\left(e_{j}\right) \leq C\left(e_{j}\right) \quad \forall e_{j} \in E
$$

The distribution of a flow $f_{i}$ on $l$ multiple paths requires that the aggregate of the sub-flows must not exceed the demand of the flow itself. Thus, we have

$$
\Sigma_{l} f_{i_{l}} \leq f_{i} \quad \forall f_{i}
$$

\subsubsection{Objective function}

The objective of the optimization model is to achieve maximum per-flow throughput. It states that the sum of flow out of source to all available parallel paths is maximized. Thus we have

$$
\operatorname{Max} \quad \Sigma_{f_{i}} \Sigma_{e_{j} \in E} f_{i} * T_{f_{i}}\left(e_{j}\right)
$$

The objective function along with the complete set of constraints is listed in Equations 1 to 9.

$$
\begin{aligned}
& \operatorname{Max} \\
& \qquad \Sigma_{f_{i}} \Sigma_{e_{j} \in E} f_{i} * T_{f_{i}}\left(e_{j}\right) \\
& \text { s.t. } \\
& \Sigma_{f_{i}} f_{i} * T_{f_{i}}\left(e_{j}\right) \leq C\left(e_{j}\right) \quad \forall e_{j} \in E \\
& \Sigma_{n_{k} \in N} f_{i} * T_{f_{i}}\left(e_{\left(n_{s_{i}}, n_{k}\right)}\right)=\Sigma_{n_{p} \in N} f_{i} * T_{f_{i}}\left(e_{\left(n_{p}, n_{\mathrm{d}_{i}}\right)}\right) \quad \forall f_{i} \\
& f_{i} * T_{f_{i}}\left(e_{\left(n_{j}, n_{k}\right)}\right)=f_{i} * T_{f_{i}}\left(e_{\left(n_{k}, n_{p}\right)}\right) \\
& \forall n_{k} \in N \backslash\left\{v_{s_{i}}, v_{d_{i}}\right\}, \forall f_{i}, n_{j} \neq n_{p} \\
& \Sigma_{e_{j} \notin P\left(f_{i}\right)} f_{i} * T_{f_{i}}\left(e_{j}\right)=0 \\
& \Sigma_{l} f_{i_{l}} \leq f_{i} \quad \forall f_{i} \\
& \forall f_{i}
\end{aligned}
$$




$$
\begin{aligned}
& \Sigma_{f_{i}} f_{i} * T_{f_{i}}\left(e_{j}\right)+\Sigma_{f_{i}} \Sigma_{e_{k}} f_{i} * T_{f_{i}}\left(e_{k}\right) \leq C\left(e_{j}\right) \\
& \forall e_{k} \in L_{\mathrm{CO}}\left(e_{j}\right) \\
& \Sigma_{f_{i}} f_{i} * T_{f_{i}}\left(e_{j}\right) \leq C\left(e_{j}\right)-\Sigma_{f_{i}} \Sigma_{e_{k}} \operatorname{demand}_{f_{i}}\left(e_{k}\right) \\
& \forall e_{k} \in L_{\mathrm{nCO}}\left(e_{j}\right) \\
& \Sigma_{e_{k} \in P\left(f_{i}\right)} T_{f_{i}}\left(e_{k}\right)=0 \quad \forall f_{i}, \forall e_{k} \in L_{\mathrm{nCO}}\left(e_{j}\right) \exists e_{l} \in L_{\mathrm{nCO}}\left(e_{k}\right)
\end{aligned}
$$

\subsection{Model validation}

Optimization models are NP-hard and cannot be solved to give generic solution. However, for specific network instances, global optimum can be achieved using numerical solvers. We have used LPSolve and programmed the model using AMPL language. The values of the variables $T_{f_{i}}\left(e_{j}\right), \forall e_{j} \in E, \forall f_{i}$ achieved by the numerical solution provide the routing information. For each flow, the chain of links originating from source with non-zero value of $T_{f_{i}}\left(e_{j}\right)$ forms a distinct path to destination. The actual value of the variables is the normalized amount of data to be routed through a particular path.

To validate the effectiveness of the optimization model in accurately predicting end-to-end throughput and accuracy of incorporated interference model in capturing the impact of interference, we replicate the network scenario (Manhattan network) in Opnet Simulator and compare the results achieved through numerical solution of the model and the Simulator. We have compared per-flow throughput achieved using the model with that of Opnet simulations. We have also observed the impact of constant and variable node density (resulting in increased coordinated and non-coordinated interference respectively) on average network throughput for the model and simulation results to test the effectiveness of constraints for coordinated and non-coordinated interference.

We use a 100-node network with nodes arranged in a $10 \times 10$ grid, such that each node can communicate with four neighboring nodes to its left, right, top, and bottom. Forty source-destination pairs have been selected randomly. All source nodes generate a load of $2 \mathrm{Mbps}$ CBR traffic, which ensures that the network operates under saturated traffic load. Multipath routing for Opnet is achieved by statically adding the routes that have been achieved through solution to optimization model for the specific network instance. Figure 3a compares per-flow throughput achieved using the model and the simulations. The flows are sorted in increasing order of achieved end-to-end throughput. The bar graph shows a good match of the model results with simulations. The average

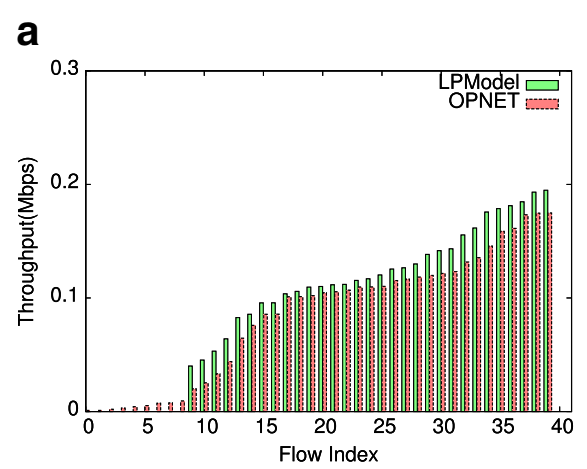

b

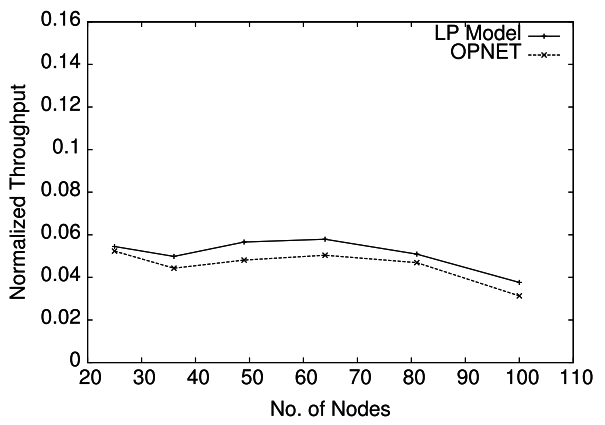

C

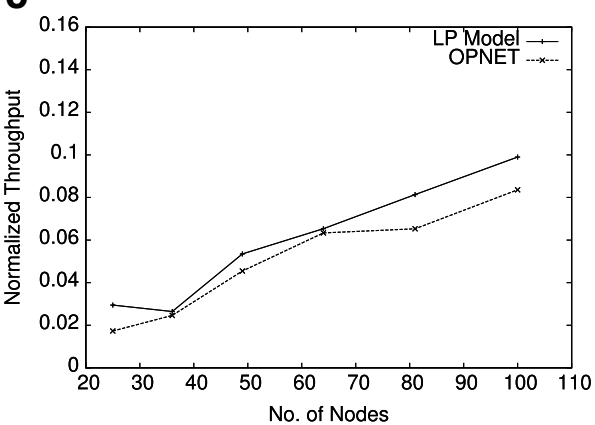

Figure 3 Optimization model validation. (a) Per-flow throughput comparison of optimization model and Opnet Simulation for Manhattan network. (b) Impact of variable node density (increased coordinated interference) on average throughput. (c) Impact of constant node density (variable number of nodes and non-coordinated interference) on average flow throughput. 
per-flow throughput achieved using simulation is $\approx 89 \%$ of the average per-flow throughput achieved using the model.

The second set of experiments is performed to test the effectiveness of model for different levels of coordinated and non-coordinated interference. This is achieved by increasing the number of nodes in the network with (i) constant terrain dimensions $\left(120 \times 120 \mathrm{~m}^{2}\right)$, which results in increased coordinated interference but relatively constant non-coordinated interference and (ii) proportionately increasing terrain dimensions, which results in increased non-coordinated interference. We have simulated the Manhattan topology with 25, 36, 49, 64, 81, and 100 nodes.

In case of constant terrain dimensions, we have generated the aggregate traffic load of $80 \mathrm{Mbps}$ from multiple sources for all scenarios. Increasing the load beyond this value does not result in increased throughput for all cases. Figure $3 \mathrm{~b}$ compares normalized (against $80 \mathrm{Mbps}$ ) aggregate end-to-end throughput achieved using the model with the simulations for constant terrain dimensions case. It is observed that the throughput slightly decreases as the number of nodes are increased. This is because of the increased coordinated interference that results in increased transmission losses. It can also be observed that the model predicts the throughput of the network within $90 \%$ of the throughput achieved using simulations.

In case of variable terrain dimensions, we have used 9, $12,16,16,20$, and 24 source nodes for $25,36,49,64$, 81 , and 100 nodes network, respectively. Each data source generates the traffic load of $2 \mathrm{Mbps}$, which results in saturated network load for the respective topologies. For every grid size, the experiment has been repeated five times with different randomly selected source-destination pairs and the results have been averaged. It is observed that the saturation point for different-sized networks is achieved at different values, unlike the variable node density case where all sized networks are saturated with aggregate input traffic load of 80 Mbps. This is because the network consists of more carrier sensing ranges as the network size increases. The increased carrier sensing ranges can support more traffic load. Figure 3c shows the normalized throughput (normalized against $49 \mathrm{Mbps}$, which is saturated load for 100 nodes network) of all cases. The throughput increases with the increasing network size, owing to increased number of carrier sensing ranges. The simulations throughput is $\approx 89 \%$ of the throughput achieved using numerical solution to the model. It is obvious that the accuracy of model is not affected by the increase in coordinated or non-coordinated interference. Therefore, we conclude that model can effectively predict end-to-end throughput for multihop wireless networks.

\section{AMRTC - adaptive multipath routing with topology control}

The optimization model proposed above computes highquality paths for all flows. In this section, we propose a centralized topology control and greedy heuristic multipath routing scheme that can be used to compute quality paths in a given network configuration while trying to maximize the aggregate end-to-end throughput.

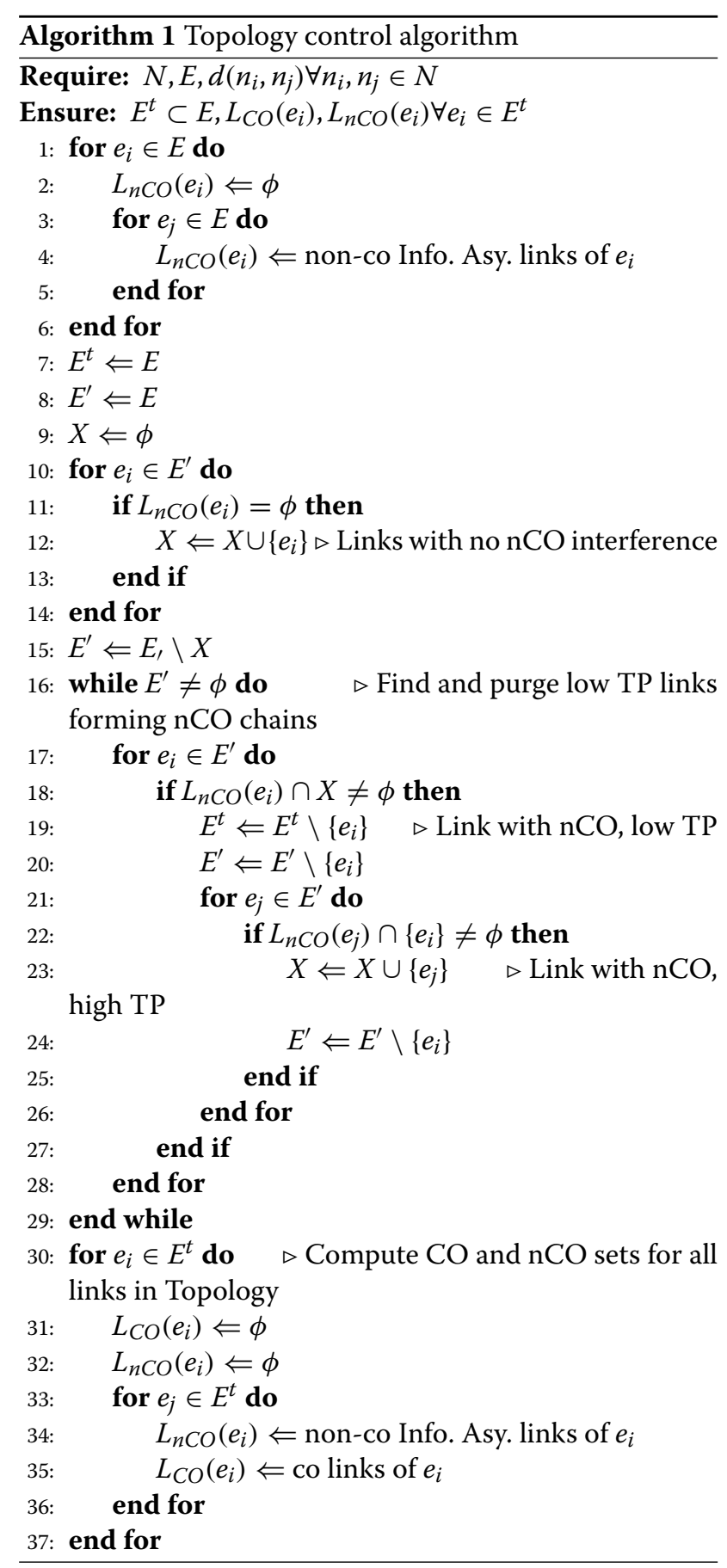


The scheme first performs topology control on the network. The links creating chain dependencies and having low channel utilization are purged from the network topology. Algorithm 1 is used for this purpose. Given the set of nodes and edges and the distance between all nodes, step 1 to step 6 of the algorithm identify non-coordinated interfering links of each link in set $E$. Step 7 to step 29 are used to purge the links that have limited throughput because of non-coordinated interference. On step 19, the link that experiences non-coordinated interference from any of the links that themselves do not experience noncoordinated interference is removed from the set $E^{t}$ of links. Removal of such links results in fewer paths for all flows; however, the forwarding capacity of remaining links will reduce negligibly (if at all). Step 23 adds to set $X$ the links that experience non-coordinated interference from the link that is to be purged from topology. Effectively, all links in set $X$ should experience no non-coordinated interference. Finally, step 30 to step 37 are used to compute the sets of coordinated and non-coordinated links for each link in the network topology (set $E^{t}$ ) after topology control.

Topology control is followed by greedy heuristic-based multipath routing algorithm. Algorithm 2 constructs one path at a time for each flow using Dijkstra algorithm [37]. The cost of using each link for Dijkstra algorithm is the inverse of the link capacity. Initially, the link capacity of all links is set to maximum. After every path construction and percentage of flow assignment on that path, the link capacity is updated by subtracting the aggregate data being forwarded by the link and its coordinated interfering links. The process is repeated until predefined maximum number of paths are constructed for each flow. Note that the first path constructed for each flow will be maximum capacity path. In each path iteration, the forwarding capacity of each subsequent path will be lesser than the already constructed paths.

AMRTC is a centralized algorithm and can be executed on any of the gateway nodes. The coordinates information is available with each deployed node. The flows in WMN are not known a priori. For this purpose, the weighted running average of the average data transmitted in previous interval and the MAC layer packet queue can be used. Higher weight may be assigned to average transmitted data to reduce the bursty nature of the flows. The information of coordinates of nodes and the flow demands can be periodically transmitted to the designated gateway through specifically designed protocol messages and pre-configured control messages only routes from nodes to the designated gateway. Any update in routing information can be communicated back to the nodes. Therefore, AMRTC can easily be transformed into a practically implementable centralized routing protocol.

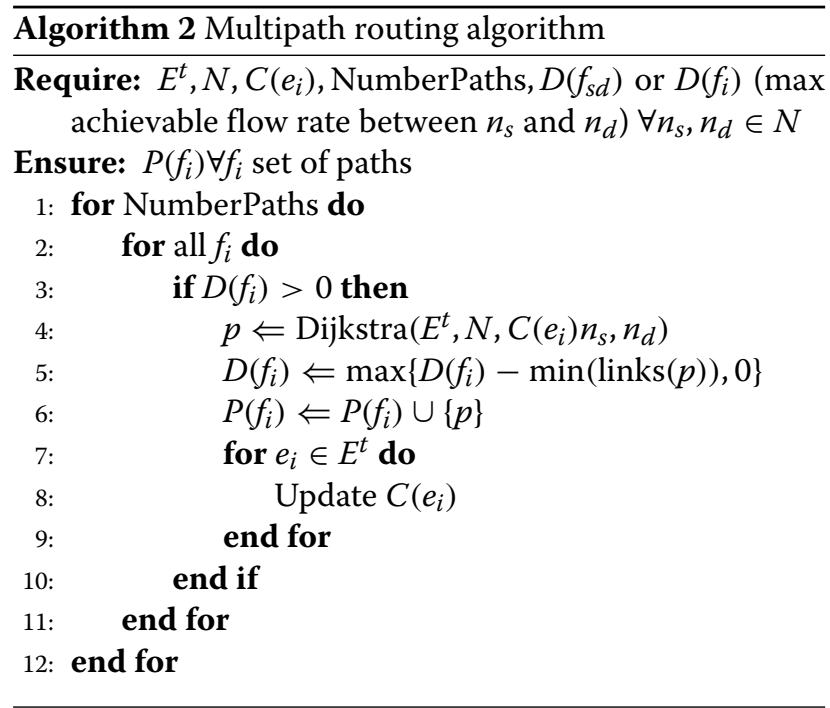

Figure 4 represents the path construction comparison of LP model and proposed AMRTC algorithm. Figure 4a shows original network topology which has been fed into LP model and AMRTC. LP model takes this network as input and constructs flow paths (Figure 4b) based on the imposed constraints to achieve maximized end-to-end throughput. As shown in the topology, the model removes low-quality links and uses remaining links to construct flow paths to achieve maximized throughput. Now consider Figure 4c representing the working of AMRTC algorithm: start with dotted arrow-headed link $(1,2)$ which is under non-CO interference of solid arrow-headed links $(10,9)$ and $(16,9)$. Working out the Topology Control phase of AMRTC algorithm, all such non-CO interfering links are identified. Then set $\mathrm{X}$ is initialized (step 12) with those links which do not experience any non-CO interference from any other link; which in this case is initially empty. The steps 16 to 29 of Algorithm 1 executes a loop on the edges to identify and remove the chains of non-CO interference. As link $(1,2)$ is on the edge of topology and does not form any chain of interference on other links, thus, it is added to set $E^{t}$ whereas links $(10,9)$ and $(16,9)$ are added to set $\mathrm{X}$ to further evaluate the interfering impact of other links on them. Now consider link $(3,10)$ which is under non-CO interference of links $(8,9)$ and $(12,13)$ and forms a chain of non-CO interference on link $(16,9)$. Thus, link $(3,10)$ is removed from the topology to break the interfering chain and links $(8,9)$ and $(12,13)$ are added to set X. Continuing with this loop, all those links are eliminated from the topology which forms interfering chains. The remaining links, stored in set $E^{t}$ and $\mathrm{X}$, experience little or no non-CO interference. Now this interferencecontrolled topology has been fed into the second phase of AMRTC which uses Dijkstra algorithm to construct paths. Figure $4 \mathrm{~d}$ represents the paths formed after complete 


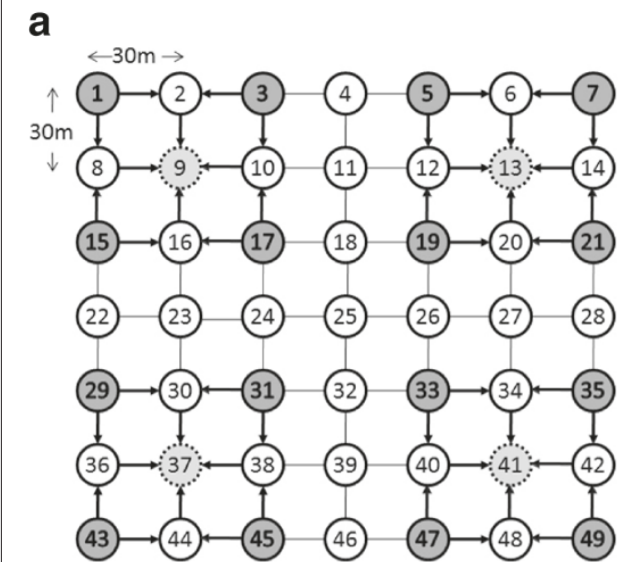

C
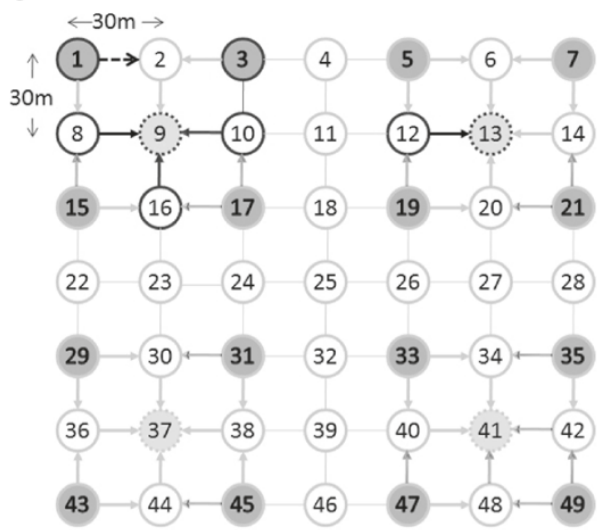

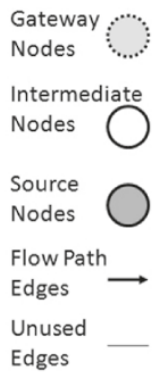

b

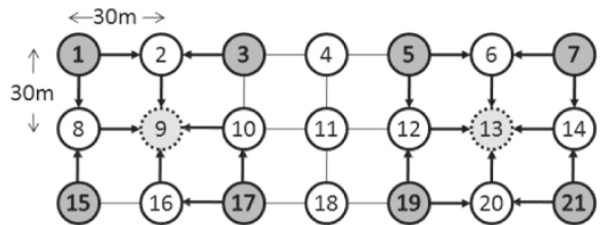

Gateway

Nodes

Intermediate

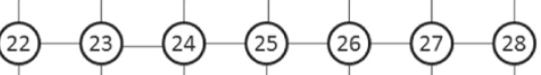

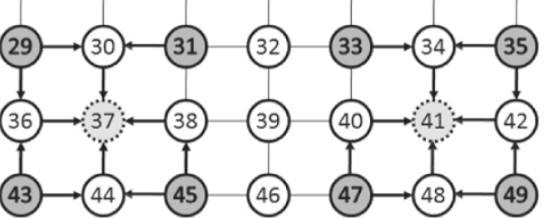

Nodes

Source

Nodes

Flow Path

Edges

Unused

Edges

d
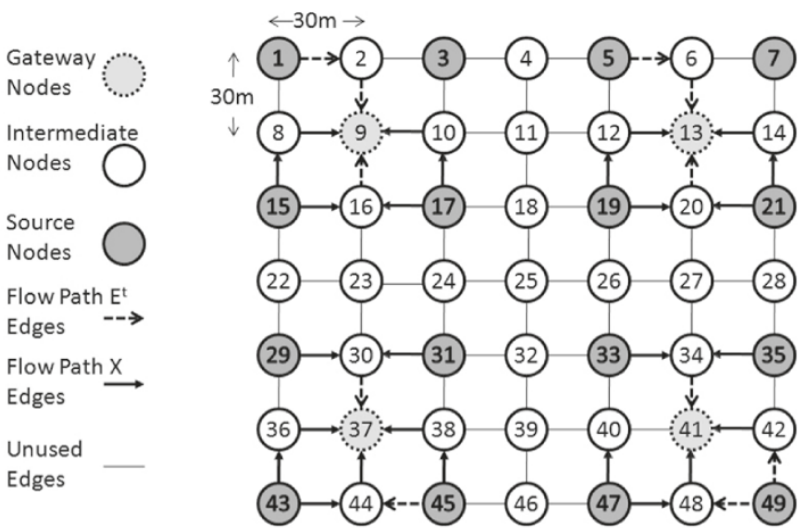

Gatew Nodes Intermediate Nodes Source Nodes Flow Path $\mathrm{E}^{\mathrm{t}}$ Edges $\rightarrow$ Flow Path $\mathrm{X}$ Edges

Unused Edges

Figure 4 LP model vs. AMRTC path construction. (a) Original topology fed into LP model and AMRTC algorithm. (b) Topology created after LP model execution. (c) Topology created with AMRTC initial run. (d) Final topology created after AMRTC complete execution.

execution of AMRTC algorithm. Compared to LP model, AMRTC constructs lesser paths for flows but considerably removes non- $\mathrm{CO}$ interfering chains to achieve better end-to-end throughput.

\section{Performance evaluations}

This section presents the simulation-based evaluation of the proposed algorithm. We have compared the performance of proposed multipath scheme AMRTC with the following three algorithms: (i) Ad hoc on-Demand Multipath Distance Vector (AOMDV) [7] (ii) Multipath Dynamic Source Routing (MDSR) [38], and (iii) AVAIL [33]. Results from the optimization model are also compared in all experiments. AOMDV, the multipath variant of well-known Ad hoc on-Demand Distance Vector, is a distance-vector routing algorithm which constructs loop-free, disjoint alternate paths in multihop networks. Similarly, MDSR is the multipath extension of DSR which uses IP source routing to construct link-wise disjoint paths from source to destination. AVAIL is a unipath routing scheme. The scheme is a modified version of Dijkstra's algorithm and discovers comparatively high throughput longer path for each flow while considering interference on links. The comparison demonstrates the effectiveness of proposed AMRTC algorithm which prioritizes quality of paths by ignoring links under severe multilevel interference.

\subsection{Experimental setup}

To evaluate the performance of the proposed algorithm, we have used Manhattan network topology [33]. In Manhattan network, the nodes are arranged in a symmetric grid such that each node is within transmission range of only its north, south, east, and west neighbors. The carrier sensing range of the nodes is set to 1.8 times the transmission range so that a node can sense the activity of all of its eight direct neighboring nodes. A set of nodes is randomly selected as source nodes to generate a load 
of $2 \mathrm{Mbps}$ each. To achieve this load, each source generates a packet of $512 \mathrm{~B}$ after every $2 \mathrm{~ms}$. Similarly, another set of nodes is randomly selected as gateway nodes to act as destinations for the source nodes. The exact number of nodes selected varies in different experiments and is mentioned with the experiment. IEEE $802.11 \mathrm{~b}$ radios are used for simulations. Table 2 summarizes the simulation parameters used in all experiments unless otherwise stated.

The evaluation is performed through four sets of experiments. In the first set, we show the effectiveness of multipath routing over unipath routing. In the second set, the performance of AMRTC is compared with AOMDV, Multipath Dynamic Source Routing (MDSR), and AVAIL in terms of offered traffic load and network throughput. The third set of experiments is conducted to compare the performance of the four algorithms with respect to quantity and length of the selected paths. The last set compares the average per-flow throughput achieved when the number of nodes are increased with constant and variable node density. This tests the algorithms under varying level of coordinated and non-coordinated interference.

\subsection{Unipath vs. multipath routing}

In this set of experiments, we have considered the 100 -node Manhattan network arranged in a $10 \times 10$ regular grid. Among these nodes, nine are randomly selected as gateway nodes while 40 source nodes are randomly selected to generate traffic towards its nearest gateway. Traffic load of the source nodes is increased from $25 \mathrm{pkts} / \mathrm{s}$ (packet transmitted every $40 \mathrm{~ms}$ ) to $1,500 \mathrm{pkts} / \mathrm{s}$ (packet transmitted every $0.6 \mathrm{~ms}$ ). Figure $5 \mathrm{a}$ plots the aggregate end-to-end throughput achieved using optimization model (multipath), AMRTC, optimization model (unipath), and $A d$ hoc on-Demand Distance Vector (AODV) (shortest path) algorithms. Results have been averaged over five simulation runs.

Table 2 Simulation parameters

\begin{tabular}{ll}
\hline Parameter & Value \\
\hline Simulation time & $1,200 \mathrm{~s}$ \\
Terrain dimensions & $500 \times 500 \mathrm{~m}^{2}$ \\
Node placement & Regular grid (Manhattan network) \\
Radio interface type & IEEE $802.11 \mathrm{~b}$ \\
Radio propagation model & Two ray \\
Propagation shadowing model & Constant (mean =4.0) \\
Application traffic & CBR \\
Packet inter-arrival time & 2 ms \\
Packet size & $512 \mathrm{~B}$ \\
\hline
\end{tabular}

It can be observed that for network load $(\approx 800$ pkts/s), performance of unipath and multipath optimization model is same. However, as the offered traffic load increases, multipath optimization model marginally outperforms unipath optimization model. The saturated traffic load that is routed by multipath-routing-based optimization model is $3.21 \mathrm{Mbps}$ while that of unipathrouting-based optimization model is $3.062 \mathrm{Mbps}$. The reason that multipath-routing-based optimization model can only marginally outperform the unipath-routingbased optimization model is attributed to the fact that even single path optimization model is able to eliminate non-coordinated interfering links. This reduces the possibility of significant number of alternate paths for the flows. On the other hand, AMRTC (saturated throughput $1.61 \mathrm{Mbps}$ ), which is multipath routing algorithm, outperforms AODV (saturated throughput 1.23 Mbps) under saturated traffic load. It can be concluded that multipath routing shall be used under all scenarios of saturated as well as unsaturated traffic load. It can also be observed that appropriately addressing the noncoordinated interference while deciding upon the routes results in good performance, even for unipath routing, which is the case for results from unipath optimization model.

\subsection{Offered traffic load and end-to-end throughput}

Next, we compare the performance of different multipath routing algorithms. The experimental setup is same as in previous section with 100 nodes arranged in grid within terrain dimensions of $120 \times 120 \mathrm{~m}^{2}$. This arrangement of nodes results in 12 neighboring nodes within the transmission range of each node and results in dense deployment of nodes. Figure $5 \mathrm{~b}$ plots the impact of increasing traffic load on aggregate end-to-end throughput when the traffic load is gradually increased from below saturation (i.e., 25 to $100 \mathrm{pkt} / \mathrm{s}$ ) point to above saturation (i.e., 200 to $1,500 \mathrm{pkt} / \mathrm{s}$ ). It is evident that AMRTC outperforms the other three schemes by achieving a higher saturated aggregate end-to-end throughput with a factor of $1.37,1.68$, and 1.63 with respect to AVAIL, AOMDV, and MDSR. This increase is the result of avoiding badly suffered links due to non-coordinated interference during path selection procedure. AMRTC outperforms AVAIL because it explicitly avoids bottleneck links as well as chain interference dependencies by purging the responsible links. AVAIL is unable to avoid the chain interference dependencies because it selects longer paths. Also note that AVAIL outperforms MDSR and AOMDV because it tries to select quality paths instead of selecting shortest paths. Therefore, chances of avoiding the bottleneck links increases in the case of AVAIL. 

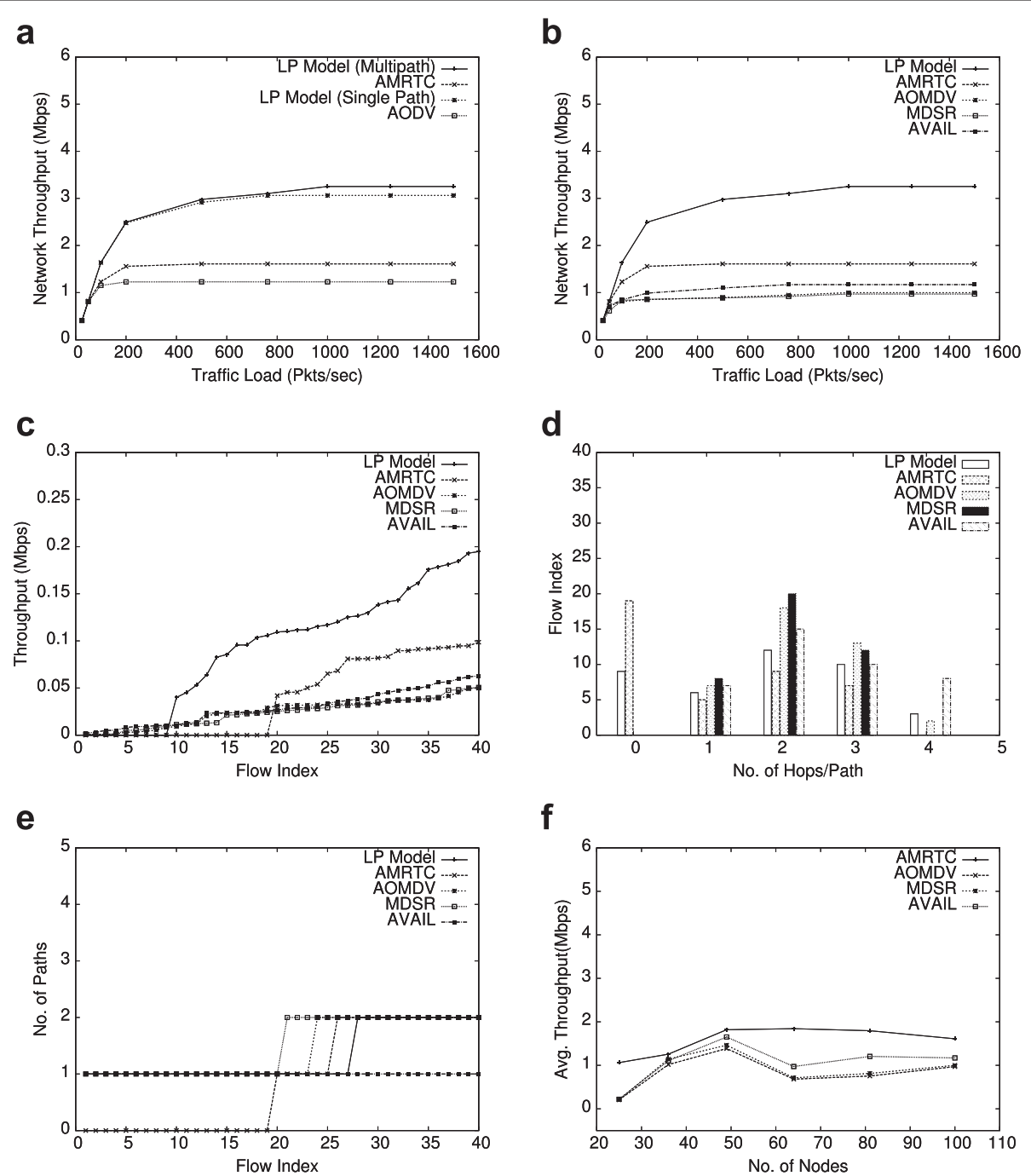

Figure 5 Performance evaluation. (a) Comparison of multiple path and single path routing. (b) Impact of increasing traffic load on throughput. (c) Per-flow throughput comparison of the four algorithms for Manhattan network. (d) Average path length comparison] (e) Number of paths comparison. (f) Impact of node density on throughput.

\subsubsection{Fairness}

Although AMRTC achieves higher aggregate end-to-end throughput compared to AVAIL, AOMDV, and MDSR, it is important for good performance of routing algorithm to provide some notion of fairness among the input flows. To evaluate the fairness, we have computed Jain's fairness index [39] for the 40 flows of 100 nodes topology. Jain's fairness indexes for flows using AMRTC, AVAIL, AOMDV, and MDSR are 0.49, 0.73, 0.73, and 0.74, respectively. This means that AMRTC is less fair when it comes to throughput distribution among flows compared to AVAIL, AOMDV, and MDSR that are equally fair. This can also be observed in Figure 5c where throughput achieved by individual flows have been plotted. It can be seen that no path has been selected for 19 flows (out of 40) by
AMRTC. To introduce fairness, we first turn to the optimization model and introduce fairness constraints in the model. We have considered following four constraints:

Proportional fair share: Every flow gets a proportion of its demand (capped by link capacity to avoid excessively large flows) fulfilled. The proportion fulfilled for all flows is within the fairness threshold ( Fair $_{\mathrm{TH}}$ ) of each other. This can be achieved using following constraint:

$\left.\mid \Sigma_{n_{k} \in N} T_{f_{i}}\left(e_{\left(n_{s_{i}}, n_{k}\right)}\right)-\Sigma_{n_{p} \in N} T_{f_{j}}\left(e_{\left(n_{s},\right.}, n_{p}\right)\right) \mid \leq \operatorname{Fair}_{\mathrm{TH}} \quad \forall f_{i}, f_{j}$

This constraint results in infeasible model for the 40 flow examples, even for large values of Fair ${ }_{\mathrm{TH}}$. This means that for the given topology, achieving this type of fairness is not 
possible. This is because of the excessive traffic load compared to the capacity of the network. Furthermore, certain flows have no feasible path that can route the amount of traffic requested.

Fair share: The demand fulfilled for all flows is within the fairness threshold of other flows. The constraint is given by following expression:

$$
\begin{aligned}
& \mid \Sigma_{n_{k} \in N} f_{i} * T_{f_{i}}\left(e_{\left(n_{s_{i}}, n_{k}\right)}\right)-\Sigma_{n_{p} \in N} f_{j} * T_{f_{j}}\left(e_{\left(n_{s_{j}}, n_{p}\right)}\right) \mid \leq \operatorname{Fair}_{\mathrm{TH}} \\
& \forall f_{i}, f_{j}
\end{aligned}
$$

This is a more strict constraint and results in infeasible model even for much smaller topologies.

Minimum demand served: This constraint ensures that for every flow, at least a minimum quantity of the flow demand, is fulfilled. The constraint is given as follows:

$$
\Sigma_{n_{k} \in N f_{i}} * T_{f_{i}}\left(e_{\left(n_{s_{i}}, n_{k}\right)}\right) \geq d_{\min } \quad \forall f_{i}
$$

This constraint can be replaced by max-min objective where the minimum demand fulfilled for any flow is maximized. It is easy to show that even for simple topology and two flows with one flow having a link that experiences information asymmetric interference, even the minimal value of $d_{\min }=128 \mathrm{kbps}$ leads to infeasible solution. This is also evident from Figure $5 \mathrm{c}$ where up to 12 flows in all algorithms receive throughput lower than $128 \mathrm{kbps}$. Given the fact that data is aggregated for multiple end users at each router, the limited throughput of $128 \mathrm{kbps}$ may not fulfill the requirements of any application. Consequently, the assigned capacity is wasted.

Maximum data limit: This constraint ensures that no flow can get the throughput more than the upper limit on demand served. The constraint is given as follows:

$$
\Sigma_{n_{k} \in N f_{i}} * T_{f_{i}}\left(e_{\left(n_{s_{i}}, n_{k}\right)}\right) \leq d_{\max } \quad \forall f_{i}
$$

This constraint produces better results compared to the above considered constraints. The results are shown in Figure 6 where 48 flows have been considered and the $d_{\max }$ is set to 1 Mbps. It can be seen that 18 flows get throughput of 1 Mbps. Note however that a number of flows still starve with no path constructed for them. The same constraint has been tested for AMRTC by limiting the flow demand and the results are shown in same figure. Three facts are worth considering that do not support the use of such a constraint in practice. First of all, limiting the maximum throughput actually results in unfair treatment of the routers that support more number of users (or fewer users with higher demand), compared to the routers that support fewer users. Secondly, it is not easy to implement the policy of limiting the data of a node, specifically in the case of infrastructure WMN where every node is an aggregation point for multiple end user devices as well as

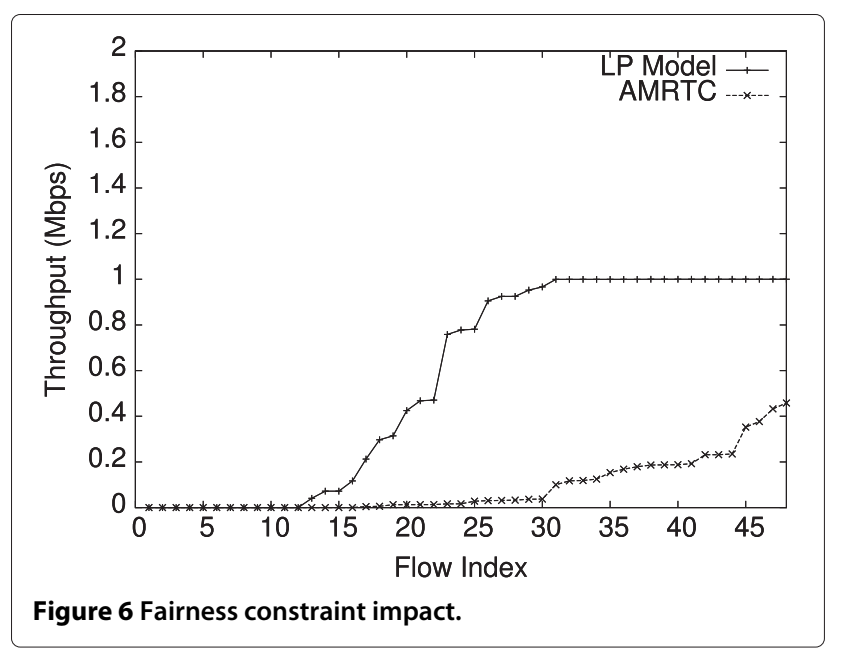

forwarding node for other flows. In such situation, it is not possible to distinguish the traffic generated from router and the traffic being forwarded for other flows. This is because all data packets have the source IP that is not same as the router itself. Finally, limiting the maximum demand fulfilled for flows still does not guarantee that other flows will not starve. At the same time, the aggregate capacity is not increased for the network.

From above discussion, it can safely be concluded that introducing the notion of fairness into the routing algorithm does not produce desired results, unless admission control algorithm is employed to limit the number of flows and the demand of each flow. Even in the presence of such measures, true fairness might not be achievable. Therefore, the presented algorithm does not attempt to incorporate fairness.

\subsection{Length and number of paths}

With experimental set of the above sections, we first compare the length of the paths selected by the four algorithms. Subsequently, we compare the average number of paths created by each algorithm. Figure $5 \mathrm{~d}$ plots the path length against the number of flows having the specified path length for the four algorithms. AMRTC selects paths with average length of 2.09. This shows that although purging a subset of links from the network results in no connectivity for certain flows, it does not significantly affect the path length for remaining flows. Longest paths are selected by AVAIL with average path length of 2.48. This is because AVAIL tries to construct longer paths with higher link diversity and improved path quality. More number of hops per path result in relatively lesser end-to-end throughput for AVAIL, compared to AMRTC. Average path length of MDSR and AOMDV is 2.1 and 2.25, respectively. This is because both algorithms 
are the shortest path algorithms and do not take quality of links into account while constructing paths. The smaller value of average path length for AOMDV and MDSR confirms that link diversity is not achieved using both algorithms, consequently leading to lower end-to-end throughput.

Figure 5e shows the number of paths created for each flow using four algorithms. The maximum number of paths for any flow constructed by four algorithms is 2 except AVAIL, which is a unipath algorithm with maximum number of paths to be 1 for all flows. The average number of paths constructed by AMRTC, AOMDV, and MDSR is $1.71,1.42$, and 1.5, respectively. AMRTC constructs fewer paths because purging of a subset of links from the network reduces the possibility of constructing multiple paths that can also result in capacity improvement. This justifies the limited improvement of multipath optimization model compared to unipath optimization model in Figure 5a. Note that AVAIL being a unipath algorithm and selecting high throughput links for each path shall have lesser interference on all links and should have achieved better end-to-end throughput; however, this is not the case. Relatively lesser throughput for AVAIL, compared to AMRTC, is attributed to the fact that the constructed paths are longer (as shown through Figure 5d). It is known that end-toend throughput exponentially decreases with increasing path length. Also note that AMRTC has constructed more paths compared to AOMDV and MDSR; however, AMRTC has outperformed MDSR and AOMDV because the selected paths have link diversity as well as low-interference links, resulting in high-quality paths. Consequently, AMRTC leads to improved end-to-end aggregate throughput because it achieves both objectives of constructing fewer paths and paths with higher-quality links.

\subsection{End-to-end delay}

End-to-end delay is another important factor for deciding the effectiveness of the routing algorithm. Figure 7 shows end-to-end delay for 100 nodes network with 40 end-to-end flows. The average per flow end-to-end delay for AMRTC (0.0437) is significantly lower, compared to AOMDV (0.0556), MDSR (0.0742), and AVAIL (0.0899). This clearly shows the advantage of selecting quality paths. With the paths having lesser collision probability, the number of unsuccessful transmissions are negligible. With the lesser number of collisions, the time is wasted because of increased backoff window as well as the time is wasted because the retransmission of packets is avoided. Therefore, minimal delay is experienced at each hop, resulting in lesser end-to-end delay for almost all flows.

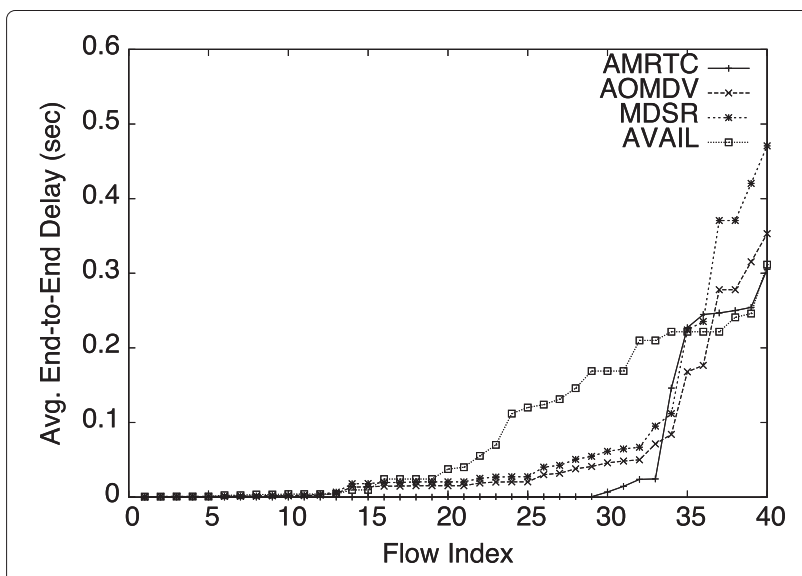

Figure 7 End-to-end delay.

\subsection{Impact of increasing node density}

The final set of experiments has been conducted to observe the impact of increasing number of nodes on average throughput using AMRTC, AVAIL, AOMDV, and MDSR. We have simulated and observed the performance of the four algorithms over 25,36, 49, 64, 81, and 100 nodes network where nodes are arranged in a regular grid. Increasing the nodes while keeping the terrain dimensions constant results in rapid increase in the number of coordinated interfering links and relatively slower increase in number of non-coordinated interfering links. On the other hand, increasing the number of nodes while keeping the node density constant (by proportionally increasing terrain dimensions) results in rapid increase of noncoordinated interference while coordinated interference remains almost constant. For example, 49 nodes network arranged in a terrain area of $120 \times 120 \mathrm{~m}^{2}$ results in 127 coordinated and 127 non-coordinated interference links. Arrangement of 100 nodes (doubling the nodes) in the same terrain area results in 203 (twofold increase) coordinated and 159 non-coordinated links. On the other hand, 100 nodes arranged in $270 \times 270 \mathrm{~m}^{2}$ terrain area result in 203 coordinated and 203 (twofold increase) noncoordinated links. We present the results with increasing number of nodes and same terrain dimensions (variable node density) as well as proportionally increased terrain dimensions (uniform node density).

Figure $5 \mathrm{f}$ shows aggregate end-to-end throughput for the four algorithms for different number of nodes deployed within constant terrain dimensions of $120 \times$ $120 \mathrm{~m}^{2}$. This results in increased node density with the increase in the number of nodes (i.e., variable node density) and leads to increased coordinated interference. It can be seen that AMRTC consistently outperforms AVAIL, AOMDV, and MDSR for all number of nodes. Also note that as node density is increased, the 
achievable aggregate end-to-end throughput by AMRTC slightly decreases. This is because of increased coordinated interference that results in minor increase in transmission losses among interfering links. The trend also suggests that creating more paths will further increase the interference and may not necessarily result in improved forwarding capacity. AVAIL is able to perform slightly better compared to AOMDV and MDSR because of link diversity.

Figure 8 shows the impact of increasing nodes with constant node density on average throughput achieved using AMRTC, AVAIL, AOMDV, and MDSR. It can be observed that AMRTC achieves significantly better throughput compared to rest of the algorithms. The improved performance is because of the adequate handling of noncoordinated interference. It can also be observed that although AVAIL avoids poor quality links, with the increase in terrain dimensions and non-coordinated interference, significant number of links experiences noncoordinated interference. AVAIL avoids all such links, resulting in longer paths that results in reduced endto-end throughput. AOMDV and MDSR achieve lesser throughput because these algorithms construct shortest paths and do not avoid bottleneck links during route selection.

\section{Conclusions}

This paper has presented an integrated approach of multipath routing and topology control to achieve throughput maximization by reducing multilevel interference dependencies and non-coordinated interference. The problem is represented as a Linear Programming model where multilevel non-coordinated interference dependencies are eliminated during the process of route selection. The model is followed by AMRTC algorithm for route

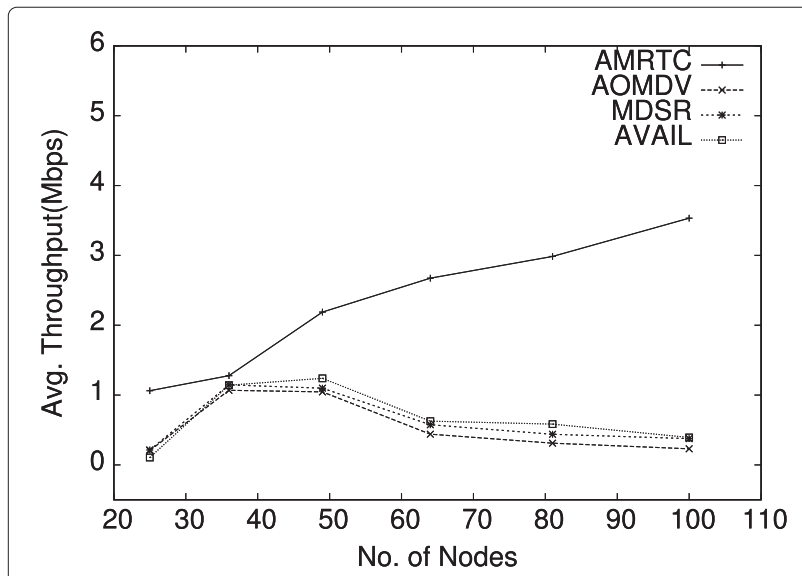

Figure 8 Impact of increasing number of nodes (with constant node density) on average throughput. selection that achieves the same objective. Eliminating non-coordinated interference dependencies also results in fewer paths, each having fewer hops. Consequently, improved end-to-end throughput is achieved, compared to the existing multipath routing algorithms as well as unipath routing algorithms that aim at constructing highquality routes.

The shortcoming of the proposed approach is that it does not ensure connectivity for all flows while eliminating multilevel non-coordinated interference. In the future, we plan to address this issue by avoiding link elimination and adjusting the flow rates of links to reduce the impact of multilevel non-coordinated interference.

\section{Competing interests}

The authors declare that they have no competing interests.

\section{Author details}

${ }^{1}$ College of EME, National University of Sciences and Technology (NUST), Islamabad, Pakistan. ${ }^{2}$ School of Electrical Engineering and Computer Science, National University of Sciences and Technology (NUST), H-12, Islamabad, Pakistan.

Received: 31 October 2013 Accepted: 23 July 2014

Published: 25 August 2014

\section{References}

1. IF Akyildiz, X Wand, W Wang, Wireless mesh networks: a survey. J. Comput. Netw. ISDN Syst. 47(4), 445-487 (2005). doi:10.1.1.61.2545

2. SMisra, SC Misra, I Woungang (eds.), Guide to Wireless Mesh Networks (Springer, New York, 2009)

3. One laptop per child. http://www.laptop.org/. Accessed 14 July 2012

4. Co-operative mobility. http://nicta.com.au/research/completed_projects/ smart_transport_and_roads/star_projects/starcom. Accessed 15 May 2014

5. Packethop. http://www.crunchbase.com/organization/packethop. Accessed 15 May 2014

6. Open Mesh/Cloud-managed wireless networks. http://open-mesh.com/. Accessed 15 May 2014

7. A Nasipuri, SR Das, On-demand multipath routing for mobile ad hoc networks, in Proceedings of International Conference on Computer Communications and Networks ICCCN, (11-13 October 1999), pp. 64-70. doi:10.1109/ICCCN.1999.805497

8. YC Hu, A Perrig, DB Johnson, Ariadne: a secure on-demand routing protocol for ad hoc networks. J. Wireless Netw. 11(1-2), 21-38 (2005). doi:10.1007//s11276-004-4744-y

9. R Draves, J Padhye, B Zill, Routing in multi-radio, multi-hop wireless mesh networks, in Proceedings of the MobiCom (Philadelphia, 26 September to 1 October 2004), pp. 114-128

10. X Meng, KTan, Q Zhang, Joint routing and channel assignment in multi-radio wireless mesh networks, in Proceedings of IEEE International Conference on Communications (Istanbul, 11-15 June 2006, vol. 9), pp. $3596-3601$

11. M Alicherry, R Bhatia, LE Li, Joint channel assignment and routing for throughput optimization in multiradio wireless mesh networks. IEEE JSAC. 24(11), 1960-1971 (2006)

12. R Banner, A Orda, Multipath routing algorithms for congestion minimization. IEEE/ACM Trans. Netw. 15(2), 413-424 (2007). doi:10.1109/TNET.2007.892850

13. S Rai, O Deshpande, C Ou, CU Martel, B Mukherjee, Reliable multipath provisioning for high-capacity backbone mesh networks. IEEE/ACM Trans. Netw. 15(4), 803-812 (2007). doi:10.1109/TNET.2007.892850

14. E Castorini, P Nobili, C Triki, Optimal routing and resource allocation in multi-hop wireless networks. J. Optimization Methods Softw. 23(4), 593-608 (2008). doi:10.1080/10556780801995907 
15. AB Mohanoor, S Radhakrishnan, V Sarangan, Interference aware multi-path routing in wireless networks, in Proceedings of 5 th IEEE International Conference on Mobile Ad Hoc and Sensor Systems (MASS) (Atlanta, 29 September to 2 October 2008), pp. 516-518

16. $\vee$ Raghunathan, PR Kumar, Wardrop routing in wireless networks. IEEE Trans. Mobile Comput. 8(5), 636-652 (2009). doi:10.1109/TMC.2008.164

17. W Wang, X Liu, D Krishnaswamy, Robust routing and scheduling in wireless mesh networks under dynamic traffic conditions. IEEE Trans. Mobile Comput. 8(12), 1705-1717 (2009). doi:10.1109/TMC.2009.86

18. A Capone, G Carello, I Filippini, S Gualandi, F Malucelli, Routing, scheduling and channel assignment in wireless mesh networks: optimization models and algorithms. J. Ad Hoc Netw. 8(6), 545-563 (2010). doi:10.1016/j.adhoc.2009.11.003

19. X Wang, JJ Garcia-Luna-Aceves, Embracing interference in ad hoc networks using joint routing and scheduling with multiple packet reception. J. Ad Hoc Netw. 7(2), 460-471 (2009). doi:10.1016/j.adhoc.2008.05.001

20. V Ramamurthi, A Reaz, D Ghosal, S Dixit, B Mukherjee, Channel, capacity, and flow assignment in wireless mesh networks. J. Comput. Netw. 55(9), 2241-2258 (2011). doi:10.1016/j.comnet.2011.03.007

21. F Javadi, KS Munasinghe, A Jamalipour, Rapid and reliable routing mesh protocol (RRRMP), in Proceedings of IEEE International Conference on Communications (ICC) (Capetown, 2010), pp. 1-5

22. F Kandah, W Zhang, Y Singh, J Li, Interference-aware robust wireless mesh network design, in Proceedings of IEEE Global Telecommunications Conference (GLOBECOM) (Miami, 6-10 December 2010), pp. 1-5

23. Z Ning, L Guo, Y Peng, X Wang, Joint scheduling and routing algorithm with load balancing in wireless mesh network. J. Comput. Electrical Eng. 38(3), 533-550 (2012). doi:10.1016/j.compeleceng.2011.12.001

24. DD Couto, D Aguayo, J Bicket, R Morris, A high throughput path metric for multihop wireless routing, in Proceedings of the Mobicom (California, 14-19 September 2003), pp. 134-146

25. Y Yang, J Wang, R Kravets, Designing routing metrics for mesh networks, in Proceedings of IEEE Workshop on Wireless Mesh Networks (WiMesh) (Santa Clara, 2005)

26. H Aiache, V Conan, L Lebrun, S Rousseau, Laett: a load dependent metric for balancing internet traffic in wireless mesh networks, in Proceedings of IEEE International Conference on Mobile Ad Hoc and Sensor Systems (MASS) (Atlanta, 29 September to 2 October 2008), pp. 629-634

27. W Jiang, S Liu, Y Zhu, Z Zhang, Optimizing routing metrics for large-scale multi-radio mesh networks, in Proceedings of International Conference of Wireless Communications, Networking and Mobile Computing (WiCom'07) (Shanghai, 21-25 September 2007), pp. 1550-1553

28. H Hassanein, A Zhou, Routing with load balancing in wireless ad hoc networks, in Proceedings of 4th ACM International Workshop on Modeling, Analysis and Simulation of Wireless and Mobile Systems (MSWiM '01) (Rome, 16-21 July 2001), pp. 89-96

29. DM Shila, T Anjali, Load-aware traffic engineering for mesh networks, in Proceedings of 16th International Conference of Computer Communications and Networking (ICCCN'07) (Honolulu, 13-16 August 2007), pp. 1040-1045

30. AP Subramanian, MM Buddhikot, S Miller, Interference aware routing in multi-radio wireless mesh networks, in Proceedings of 2nd IEEE Workshop on Wireless Mesh Networks (WiMesh '06) (Reston, 25 September 2006), pp. 55-63

31. M Kumar, N Kumar, RB Patel, Capacity and interference aware link scheduling with channel assignment in wireless mesh networks. J. Netw Comput. Appl. 34(1), 30-38 (2011). doi:10.1016/j.jnca.2010.10.001

32. M Garetto, T Salonidis, EW Knightly, Modeling per-flow throughput and capturing starvation in CSMA multihop wireless networks. IEEE/ACM Trans. Netw. 16(4), 864-877 (2008). doi:10.1109/TNET.2007.902687

33. T Salonidis, M Garetto, A Saha, E Knightly, Identifying high throughput paths in 802.11 mesh networks: a model-based approach, in Proceedings of IEEE International Conference on Network Protocol (ICNP'07) (Beijing, 16-19 October 2007), pp. 21-30

34. S Razak, V Kolar, NB Abu-Ghazaleh, A MAC interaction aware routing metric in wireless networks, in Proceedings of Proceedings of the 13th ACM International Conference on Modeling, Analysis, and Simulation of Wireless and Mobile Systems (MSWiM'10) (Bodrum, 17-21 October 2010), pp. 118-121

35. S Razak, NB Abu-Ghazaleh, Self-interference in multi-hop wireless chains: geometric analysis and performance study, in Proceedings of the 7th
International Conference on Ad-hoc, Mobile and Wireless Networks (ADHOC-NOW'08) (Sophia-Antipolis, 10-13 September 2008, vol. 5198), pp. $58-71$

36. S Nelakuditi, ZL Zhang, On selection of paths for multipath routing, in Proceedings of Proceedings of the 9th International Workshop on Quality of Service (IWQOS '01) (Karlsruhe, 6-8 June 2001), pp. 170-186

37. EW Dijkstra, A note on two problems in connexion with graphs. J. Numerische Mathematik. 1(1), 269-271 (1959). doi:10.1007/BF01386390

38. MK Marina, SR Das, On-demand multi path distance vector routing in ad-hoc networks, in Proceedings of Ninth International Conference on Network Protocols (ICNP'01) (Riverside, 11-14 November 2001), pp. 14-23

39. M Hassan, R Jain, High Performance TCP/IP Networking. (Pearson/Prentice Hall, New Jersey, 2004)

doi:10.1186/1687-1499-2014-140

Cite this article as: Iqbal et al:: Interference-aware multipath routing in wireless mesh network. EURASIP Journal on Wireless Communications and Networking 2014 2014:140.

\section{Submit your manuscript to a SpringerOpen ${ }^{\circ}$ journal and benefit from:}

- Convenient online submission

- Rigorous peer review

- Immediate publication on acceptance

- Open access: articles freely available online

- High visibility within the field

- Retaining the copyright to your article

Submit your next manuscript at $>$ springeropen.com 